\title{
ROLE OF SYSTEMIC THERAPY IN THE DEVELOPMENT OF LUNG SEQUELAE AFTER CONFORMAL RADIOTHERAPY IN BREAST CANCER PATIENTS
}

\author{
Zoltán Varga, Ph.D., * Adrienn Cserháti, M.D.,* Gyöngyi Kelemen, M.D., * \\ Krisztina Boda, Ph.D., ${ }^{\dagger}$ László Thurzó, M.D., Ph.D., ${ }^{*}$ and Zsuzsanna Kahán, M.D., Ph.D.* \\ Departments of * Oncotherapy and ${ }^{\dagger}$ Medical Informatics, University of Szeged, Szeged, Hungary
}

\begin{abstract}
Purpose: To analyze the risk of radiogenic lung damage in breast cancer patients after conformal radiotherapy and different forms of systemic treatment.

Methods and Materials: In 328 patients receiving sequential taxane-based chemotherapy, concomitant hormone therapy (tamoxifen or aromatase inhibitors), or no adjuvant systemic therapy, symptomatic and asymptomatic lung sequelae were prospectively evaluated via the detection of visible CT abnormalities, 3 months or 1 year after the completion of the radiotherapy.

Results: Significant positive associations were detected between the development of both pneumonitis and fibrosis of Grade 1 and patient age, ipsilateral mean lung dose, volume of the ipsilateral lung receiving $20 \mathrm{~Gy}$, and irradiation of the regional lymph nodes. In multivariate analysis, age and mean lung dose proved to be independent predictors of early (odds ratio $[\mathrm{OR}]=1.035,95 \%$ confidence interval $[\mathrm{CI}] 1.011-1.061$ and $\mathrm{OR}=1.113,95 \% \mathrm{CI}$ 1.049-1.181, respectively) and late $(O R=1.074,95 \%$ CI 1.042-1.107 and $O R=1.207,95 \%$ CI 1.124-1.295, respectively) radiogenic lung damage, whereas the role of systemic therapy was significant in the development of Grade 1 lung fibrosis $(p=0.01)$. Among the various forms of systemic therapy, tamoxifen increased the risk of late lung sequelae $(\mathrm{OR}=\mathbf{2 . 4 4 2}, 95 \% \mathrm{CI} \mathbf{1 . 1 2 0}-\mathbf{5 . 3 2 6}, p=\mathbf{0 . 0 2 5})$. No interaction was demonstrated between the administration of systemic therapy and the other above-mentioned parameters as regards the risk of radiogenic lung damage. Conclusions: Our analyses demonstrate the independent role of concomitant tamoxifen therapy in the development of radiogenic lung fibrosis but do not suggest such an effect for the other modes of systemic treatment. (c) 2011 Elsevier Inc.
\end{abstract}

Radiation lung sequelae, Tamoxifen, Aromatase inhibitors, Taxanes.

\section{INTRODUCTION}

The various forms of adjuvant therapy, including postoperative irradiation and systemic therapy in breast cancer, contribute to the decreasing mortality rate among the affected population $(1,2)$. Adjuvant radiotherapy is a standard form of treatment after breast-conserving surgery and is sometimes also practised after mastectomy (3, 4). Nonetheless, radiotherapy might cause long-term toxicity, such as radiation-induced pneumonitis and fibrosis of the lung. Early radiation-induced symptoms arise within 6 months after the completion of radiotherapy and may later progress to a chronic fibrotic status $(5,6)$. The incidence of radiationinduced lung injury in breast cancer in different prospective studies varies between $4.5 \%$ and $63 \%$ (7-15). Most radiation pneumonitis and fibrosis are asymptomatic after breast radiotherapy because of the relatively small irradiated lung volume and low radiation dose. Even so, the prevention of radiation lung damage can be facilitated by the identification of risk factors. Patient age, irradiated volume of the lung, and the dose to it are clearly related to early and late radiation damage $(7,9,13-15)$. Our earlier studies suggested the synergistic effect of lung dose with age in patients older than 59 years (13).

For the postoperative treatment of breast cancer, internationally accepted guidelines exist, based on decades of clinical experience $(16,17)$. Adjuvant systemic therapies roughly halve the risk of death and contribute to local control. The application of the various forms of endocrine therapy or chemotherapy is more and more individualized on the basis of the features of the tumor. The different hormone therapy options are widely applied in hormone receptor-positive breast cancers, either during or after radiotherapy $(16,17)$. Tamoxifen, a competitive antagonist of 17- $\beta$-estradiol, is used in both premenopausal and postmenopausal patients, whereas the aromatase inhibitors
Reprint requests to: Zsuzsanna Kahán, M.D., Ph.D., Department of Oncotherapy, University of Szeged, Korányi fasor 12, H-6720 Szeged, Hungary. Tel: (+36) 62-545406; Fax: (+36) 62-545922; E-mail: kahan@onko.szote.u-szeged.hu

Conflict of interest: none.
Acknowledgment-The authors thank Dr. Zsuzsanna Várnay for participating in the double reading of the CT scans.

Received Dec 18, 2009, and in revised form March 13, 2010. Accepted for publication March 17, 2010. 
that efficiently block the synthesis of estrogens are given exclusively to postmenopausal patients. Numerous chemotherapy regimens are used, and because the inclusion of paclitaxel or docetaxel has significantly improved efficacy, these taxane-based regimens now represent the standard of care in high-risk cases. In some reports, the simultaneous administration of tamoxifen during radiotherapy was associated with the development of early or late radiation lung damage (18-20), whereas no such relationship was observed in other studies $(9,14,21-23)$. No similar data have been published to date regarding the use of aromatase inhibitors during breast radiotherapy, except for one small study (24). Nevertheless, Azria et al. (25) and Ozsahin et al. (26) reported that the increased level of subcutaneous fibrosis after radiotherapy in the randomized COHORT study was not related to the simultaneously administered letrozole therapy but rather to the low radiation-induced CD8 lymphocyte apoptosis (RILA) status of the patient $(25,26)$. Concomitantly administered radiochemotherapy leads to unacceptable acute and late lung toxicity (27-29). The sequential use of chemotherapy including the taxane-based regimens and irradiation does not seem to increase the risk of lung injury $(9,11,30)$.

\section{METHODS AND MATERIALS}

The study was approved by the institutional review board of the University of Szeged, and all enrolled patients gave their written informed consent to participate in the study.

\section{Study population}

Between November 2001-August 2004 and January 2006-May 2008, patients after curative surgery for breast cancer who required radiotherapy were recruited at our department. Patients with prior malignancy, pulmonary or autoimmune disease, or any other significant health problem, or who were receiving glucocorticoid therapy, were excluded. The initial surgery was either mastectomy or breastconserving surgery, with sentinel lymph node biopsy or/and axillary lymph node dissection. The patient and tumor characteristics are presented in Table 1. Data were collected on smoking habits, with participants categorized as past or present smokers or nonsmokers.

\section{Systemic treatment}

Depending on the systemic treatment administered, the subjects were subdivided postoperatively into four groups: 79 patients completed a taxane-based perioperative chemotherapy regimen (involving either docetaxel at a dose of $75 \mathrm{mg} / \mathrm{m}^{2}$ or paclitaxel at a dose of 175 or $200 \mathrm{mg} / \mathrm{m}^{2}$ in combination or sequentially with doxorubicin at a dose of $60 \mathrm{mg} / \mathrm{m}^{2}$ or epirubicin at a dose of $75 \mathrm{mg} / \mathrm{m}^{2}$ and cyclophosphamide at a dose of $800 \mathrm{mg} / \mathrm{m}^{2}$ ) for $\geq 4$ weeks before radiotherapy (taxane group); 159 patients received adjuvant hormonal therapy: tamoxifen, $20 \mathrm{mg} / \mathrm{day}, n=77$, or aromatase inhibitor (anastrozole, $1 \mathrm{mg} / \mathrm{day}$, or letrozole, $2.5 \mathrm{mg} / \mathrm{day}) n=82$, started $\geq 2$ weeks before the initiation of radiotherapy (tamoxifen and aromatase inhibitor groups, respectively); and a further 90 patients (control group) received no systemic medication before, during, or after the radiotherapy.

\section{Radiotherapy}

Computed tomography-based three-dimensional treatment planning and conformal radiotherapy was in all cases performed with the patient in a supine position. All relevant technical details have been published previously $(13,31)$. Briefly, CT images were acquired at every $1 \mathrm{~cm}$ throughout the entire planning volume. The target volume and organs at risk were contoured on the CT slices in the radiotherapy planning system. The planning target volume coverage was analyzed via the dose-volume histograms and isodose visualization. Local (operated breast or chest wall) or locoregional (the former together with coverage of any of the following regions: axillary, supraclavicular, and internal mammary lymph nodes) radiotherapy was chosen according to the local protocol. A standard technique of irradiation was used to cover the operated breast/chest wall and the internal mammary lymph nodes with tangential fields, and from January 2005, individually weighted 6- or 15-MV segmental fields were superimposed on the tangential fields, using a multileaf collimator for better dose homogeneity. The axillary and supraclavicular nodes were irradiated with a direct photon field. The tumor bed boost was delivered with either 6-MV photon or $8-15-\mathrm{MeV}$ electron fields. The radiation dose to the remaining breast parenchyma/chest wall and to the lymph nodes, if indicated, was $25 \times 2$ Gy (prescribed to the mean of the planning target volume); a tumor bed boost of 5-8 $\times 2$ Gy was delivered when necessary. Organ-at-risk constraints were used as previously described, and the volume of the ipsilateral lung receiving more than $20 \mathrm{~Gy}\left(\mathrm{~V}_{20 \mathrm{~Gy}}\right)$ and the mean lung dose (MLD) were registered for the purpose of this study $(13,31)$. Radiotherapy was delivered with a linear accelerator in 5 fractions per week. Although the technical background changed due to modernization in 2005, the use of different planning and positioning systems or field-shaping techniques did not influence the radiotherapy protocol significantly.

\section{Evaluation of radiogenic lung damage}

At 3 months and at 1 year after the completion of the radiotherapy, clinical follow-up visits with special attention to pulmonary symptoms (fever, cough, and dyspnea) and diagnostic CT examinations were performed. The CT scans at these stages were compared with those provided for radiotherapy planning purposes according to the accepted criteria (13). The evaluation was performed independently by two physicians. The categories of Grade 1 pneumonitis or Grade 1 fibrosis were used to describe the new appearance of inflammatory or fibrotic abnormalities in the radiation fields at the two time points, regardless of whether the patient simultaneously developed specific clinical signs and symptoms, according to the Common Toxicity Criteria version 2.0. The CT examinations were not performed 1 year after the radiotherapy in 15 cases because of the progression of the breast cancer or some other disease $(n=9)$, the lack of compliance of the patient $(n=5)$, or a car accident $(n=1)$.

\section{Statistical analysis}

The various patient- and radiotherapy-related characteristics were examined in the four groups of patients according to the presence or absence of radiogenic lung damage by univariate statistical methods, with one-way analysis of variance and $\chi^{2}$ test being used for continuous and categoric variables, respectively. The relationships of age and MLD were examined by analysis of covariance. The associations between severity of radiation lung damage and patient age were analyzed by independent-samples $t$ test.

Logistic regression models were applied to examine the potential risk factors for the occurrence of early and late $\mathrm{CT}$ changes with or 
without clinical symptoms. First, binary univariate logistic regression models were used separately, followed by the multivariate logistic regression model to examine the joint effects and interactions. A stepwise procedure was used with a likelihood ratio test. The software program SPSS 15.0 for Windows (SPSS, Chicago, IL) was applied for statistical analysis.

\section{RESULTS}

Altogether 328 patients were enrolled into the study. The main characteristics of the patients who participated are listed in Table 1 . The mean $( \pm \mathrm{SE})$ age of the study population was $59.4 \pm 0.6$ years (range, 28.2-87.1 years). The vast majority (96\%) of the tumors were invasive, and two thirds were invasive ductal cancers. All the patients in the hormone therapy groups had estrogen and/or progesterone receptor-positive tumors. The HER2 status did not differ significantly in the different groups. The distribution of the irradiated volumes among the four groups, together with other radiotherapy-related data, is presented in Table 1. The rate of locoregional radiotherapy, and as a consequence the MLD and $V_{20 G y}$, were significantly higher in the taxane group $(p<0.001)$ (Table 1). The proportion of past or present smokers was the highest in the tamoxifen group ( $p=0.052$ ). Radiation pneumonitis of Grade 1 was found in $41.8 \%$ of the patients, and $5.8 \%$ had mild clinical symptoms. Grade 1 radiation fibrosis developed in $30.4 \%$ of the patients; none of them had symptoms. The incidence of radiation pneumonitis or fibrosis did not exhibit significant variations during the study.

The presence of early or late radiogenic lung damage was compared with the various patient- and radiotherapy-related characteristics (Tables 2 and 3). Highly significant associations were found between the presence of early or late radiogenic lung changes and patient age, MLD, and $\mathrm{V}_{20 \mathrm{~Gy}}$. There was a weak negative correlation between age and MLD in the overall study population $(r=-0.143, p=0.009)$. Nodal irradiation favored lung damage ( $p=0.017$ at 3 months, and $p<$ 0.001 at 1 year after the radiotherapy). One year after radiotherapy, Grade 1 fibrosis was more frequent when mastectomy had been performed $(p<0.001)$ (Table 3$)$, though this was probably a consequence of the higher frequency of supraclavicular and axillary irradiation after mastectomy than after tumor excision $(49.6 \%$ vs. $32.6 \%$, respectively, $p<0.003$ ). A past or present history of smoking did not influence the degree of radiogenic lung damage 3 months and 1 year after radiotherapy (Tables 2 and 3).

The incidence of Grade 1 pneumonitis or Grade 1 fibrosis did not differ in the four treatment groups, but most cases of symptomatic pneumonitis were observed in the tamoxifen group $(p=0.076)$ ) (Table 4). When the effect of patient age on the radiogenic lung changes was analyzed in the different treatment groups, the patients with symptomatic pneumonitis in the tamoxifen group proved to be significantly older than the patients without lung damage $(p=0.013)$ (Fig. 1). A significant association was found between the presence of Grade 1 pneumonitis and the presence of Grade 1 fibrosis $(p<0.001$, McNemar test).

\section{Univariate analysis}

To estimate the risks of pneumonitis or fibrosis, the effects of patient age, MLD, and the different modes of systemic treatment were first studied in binary univariate logistic regression models. The risks of Grade 1 pneumonitis and Grade 1 fibrosis were increased 3 months and 1 year, respectively, after radiotherapy, with odds ratio $(\mathrm{OR})=1.030(95 \%$ confidence interval [CI] 1.009-1.051, $p=0.004)$ and $\mathrm{OR}=1.054$ (95\% CI 1.029-1.081, $p<0.001$ ), respectively, for every 1 year increase in the age of the patient. Significant positive associations were demonstrated between the risk of Grade 1 pneumonitis and MLD (OR $=1.080 ; 95 \%$ CI 1.027-1.135, $p=0.003)$ and between the risk of Grade 1 fibrosis and MLD (OR $=1.156$; 95\% CI 1.091-1.224, $p<0.001)$ for every 1.0-Gy increase. Significant associations were not found between the risks of early or late radiogenic lung damage and the addition of systemic therapy (Table 4).

\section{Multivariate analysis}

The joint effects of age, MLD, the systemic treatment, and their interactions were examined in a multiple logistic

Table 1. Associations of the patient- and radiotherapy-related characteristics of the study population and the various forms of systemic therapy

\begin{tabular}{lccccc}
\hline \multicolumn{1}{c}{ Characteristic } & Control $(n=90)$ & Taxane $(n=79)$ & Tamoxifen $(n=77)$ & Aromatase inhibitor $(n=82)$ & $p$ \\
\hline $\begin{array}{l}\text { Age (y), mean } \pm \text { SE) } \\
\text { Irradiated volume }\end{array}$ & $62.4 \pm 1.0$ & $51.1 \pm 1.1$ & $56.6 \pm 1.2$ & $66.3 \pm 0.9$ & $<0.0001$ \\
$\quad$ Breast & $64(71.1)$ & $50(63.3)$ & $46(59.7)$ & $55(67.1)$ & 0.449 \\
$\quad$ Chest wall & $26(28.9)$ & $29(36.7)$ & $31(40.3)$ & $27(32.9)$ & \\
Irradiation of the regional lymph nodes & & & & \\
$\quad$ Yes & $16(17.8)$ & $66(83.5)$ & $22(28.6)$ & $22(26.8)$ & $<0.0001$ \\
$\quad$ No & $74(82.2)$ & $13(16.5)$ & $55(71.4)$ & $60(73.2)$ & $<0.0001$ \\
MLD (Gy), mean \pm SE & $8.9 \pm 0.3$ & $14.1 \pm 0.5$ & $10.7 \pm 0.5$ & $10.0 \pm 0.4$ & $<0.0001$ \\
V 20Gy (\%), mean \pm SE $_{\text {Smoking }}$ & $16.9 \pm 0.7$ & $29.0 \pm 1.1$ & $21.1 \pm 1.3$ & $19.7 \pm 1.0$ & 0.052 \\
$\quad$ Present or past smokers & $38(42.2)$ & $29(36.7)$ & $38(49.4)$ & $24(29.3)$ & \\
$\quad$ Nonsmokers & $52(57.8)$ & $50(63.3)$ & $39(50.6)$ & & \\
\hline
\end{tabular}

Abbreviations: $\mathrm{MLD}=$ mean lung dose; $\mathrm{V}_{20 \mathrm{~Gy}}=$ volume of the ipsilateral lung receiving $20 \mathrm{~Gy}$.

Values are number (percentage) unless otherwise noted. 
Table 2. Associations of patient- and radiotherapy-related characteristics of the study population and early radiation lung sequelae

\begin{tabular}{|c|c|c|c|c|c|}
\hline & \multirow[b]{2}{*}{ No change $(n=191)$} & \multicolumn{4}{|c|}{ Grade 1 pneumonitis } \\
\hline Characteristic & & Symptomatic $(n=19)$ & $p$ (vs. no change) & Any $(n=137)$ & $p$ (vs. no change) \\
\hline Irradiated volume & & & 0.086 & & 0.963 \\
\hline Breast & $66(34.6)$ & $10(52.6)$ & & $47(34.3)$ & \\
\hline Chest wall & $125(65.4)$ & $9(47.4)$ & & $90(65.7)$ & \\
\hline No & $128(67.0)$ & $9(47.4)$ & & $74(54.0)$ & \\
\hline $\operatorname{MLD}(\mathrm{Gy})$, mean $\pm \mathrm{SE}$ & $10.2 \pm 0.3$ & $12.7 \pm 1.1$ & 0.019 & $11.7 \pm 0.4$ & 0.011 \\
\hline $\mathrm{V}_{20 \mathrm{~Gy}}(\%)$, mean $\pm \mathrm{SE}$ & $20.1 \pm 0.8$ & $25.7 \pm 2.7$ & 0.024 & $23.4 \pm 0.8$ & 0.017 \\
\hline Smoking & & & & & 0.509 \\
\hline Present or past smokers & $78(40.8)$ & $5(26.3)$ & 0.232 & $51(37.2)$ & \\
\hline Nonsmokers & $113(59.2)$ & $14(73.7)$ & & $86(62.8)$ & \\
\hline
\end{tabular}

Abbreviations as in Table 1.

Values are number (percentage) unless otherwise noted.

regression model, using a stepwise algorithm. All three variables remained significant in the model (Table 5). Whereas the risks of any radiation pneumonitis and that with symptoms and the administration of systemic therapy displayed nonsignificant trends ( $p=0.080$ and $p=0.064$, respectively), the risk of fibrosis was significantly elevated by the administration of systemic therapy $(p=0.001)$ or of tamoxifen $(p=$ $0.025)$. The joint effects of age, MLD, and the systemic treatment on the risk of radiation fibrosis are illustrated in Fig. 2. Only the interaction of age and MLD remained significant in the development of late CT abnormalities (OR $=1.006 ; 95 \%$ CI $1.000-1.013, p=0.050$ with every 1 -year increase in patient age and every 1-Gy increase in MLD). No interaction was detected for the various types of systemic therapy and the dosimetric parameters, irrespective of whether the analysis extended to the entire population or only to an older age group.

\section{DISCUSSION}

Based on a complex set of clinical data on 328 breast cancer patients, this analysis revealed that the concomitant administration of tamoxifen with adjuvant radiotherapy inde- pendently increases the risk of radiation lung fibrosis, whereas the aromatase inhibitors and sequential taxanebased chemotherapy have no such effect. We believe that our findings of the advantages of conformal radiotherapy and individualized adjuvant systemic therapy make a notable contribution to the clarification of the discrepancy that has long existed regarding the relation of systemic treatment and radiation lung damage and indicate the need for the withdrawal of tamoxifen during adjuvant radiotherapy.

Tamoxifen has been widely applied for the treatment of breast cancer in the adjuvant setting, and its coadministration with adjuvant radiotherapy has been the subject of numerous studies $(7,9,14,18,19,22-24,32)$. In some of these the incidence of radiation lung complications did not differ when tamoxifen was or was not administered simultaneously with radiotherapy $(7,9,14,23,24)$. The negative results might have been due to the retrospective nature of the analyses $(14,22)$, the underpowered study populations $(9,21,24)$, the limitation of the study endpoint to pneumonitis (9), or the low sensitivity of the method of follow-up $(14,22,23)$. In other studies, the incidence of radiogenic pulmonary fibrosis proved to be significantly higher in the patients treated with tamoxifen (18-20). The

Table 3. Associations of patient- and radiotherapy-related characteristics of the study population and the late radiation lung sequelae

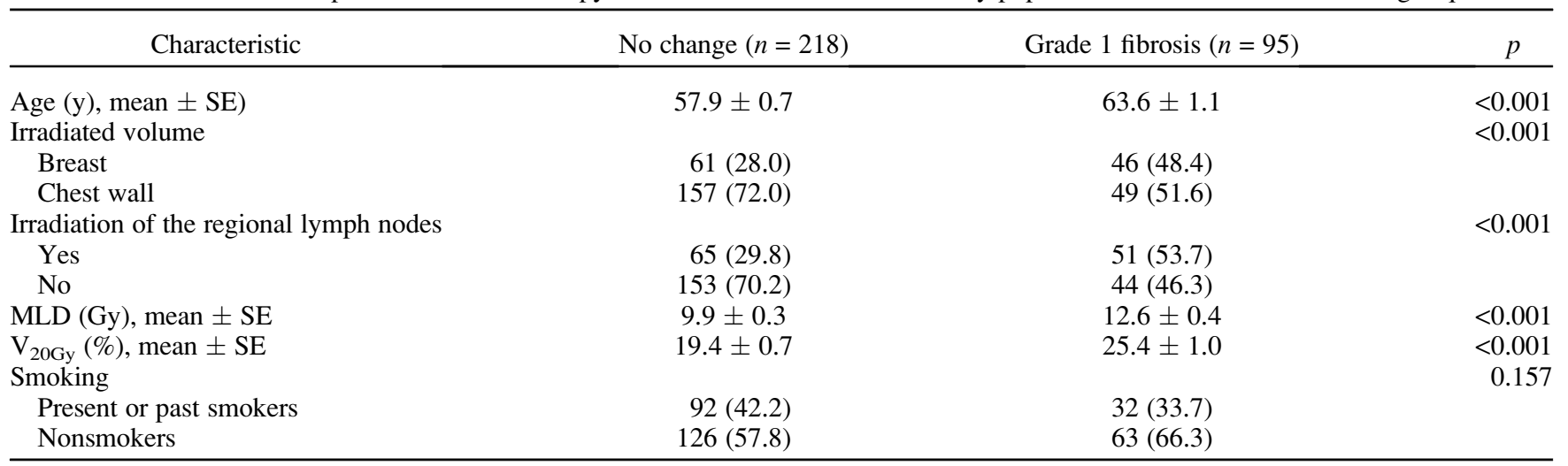

Abbreviations as in Table 1.

Values are number (percentage) unless otherwise noted. 
first such trial was that of Bentzen et al. (18): in a randomized study of 84 postmenopausal women, the risk of radiation fibrosis in the axillary and supraclavicular fields was doubled when tamoxifen was coadministered with the radiotherapy. Koc et al. (19) applied postoperative telecobalt irradiation in 111 patients and observed that regular chest CTs revealed lung fibrosis rates of $35 \%$ when tamoxifen was administered during the radiotherapy vs. $13 \%$ when it was not. Huang et al. (20) reported that tamoxifen therapy was an independent risk factor of radiation lung fibrosis when coadministered during electron-irradiation of the chest wall $(\mathrm{OR}=3.35, p=0.03)$. These three studies did not involve the use of conformal radiotherapy and could not include dose-volume histogram data in the analysis. The strength of our study is that, besides confirming tamoxifen as a risk factor in the development of radiogenic lung fibrosis, it investigated the role of systemic therapy independently of the simultaneous effects of dosimetric factors and age. These parameters can easily be taken into consideration when deciding about the radiotherapy. Our findings support and complement the well-demonstrated data on the role of tamoxifen in the development of another form of late radiogenic toxicity, subcutaneous fibrosis $(25,26,32)$. Our analysis has the limitation, however, that it does not take into account the inherent radiosensitivity of the individual. Such approaches have been suggested by the determination of circulating transforming growth factor- $\beta$ (TGF- $\beta$ ) (33$35)$ or RILA $(25,26,32)$ in breast cancer patients.

Transforming growth factor $-\beta$ is a major participant in the processes of wound healing and fibrosis, and the induction of its synthesis may speed up the vicious cycle of chemotaxis and the activation of neutrophils, $\mathrm{T}$ lymphocytes, monocytes, and fibroblasts initiated by radiotherapy (36). Tamoxifen stimulates the secretion of TGF- $\beta$ by the fibroblasts, which might serve as one explanation for the increased risk of radiation lung damage when tamoxifen is coadministered with radiotherapy. In the first 119 of our patients, we measured TGF- $\beta$ plasma levels before and during the radiotherapy, as reported earlier (13). Although we found correlations between the variations in circulating TGF- $\beta$ and both the lung density changes and the development of symptomatic pneumonitis 3 months after the radiotherapy, no differences were observed as concerns the type of systemic therapy (data not shown). There could be many reasons for this finding. First, the plasma levels of TGF- $\beta$ probably do not represent the in situ TGF- $\beta$ concentrations with adequate sensitivity (37). Second, it can not be excluded that the individual levels of drug metabolic capacity (CYP2D6 polymorphism), which determine the formation of the active metabolite of tamoxifen, as recently evidenced by clinical data (38), also influence the extent of TGF- $\beta$ induction.

We tested whether another antiestrogen, toremifen, can influence the plasma TGF- $\beta$ level. No significant change was detected in 7 metastatic breast cancer patients after treatment with toremifen (data not shown). Our results are consistent with the findings of a pilot study on metastatic breast cancer patients, whose TGF- $\beta$ plasma levels were unchanged after treatment with tamoxifen (35). 


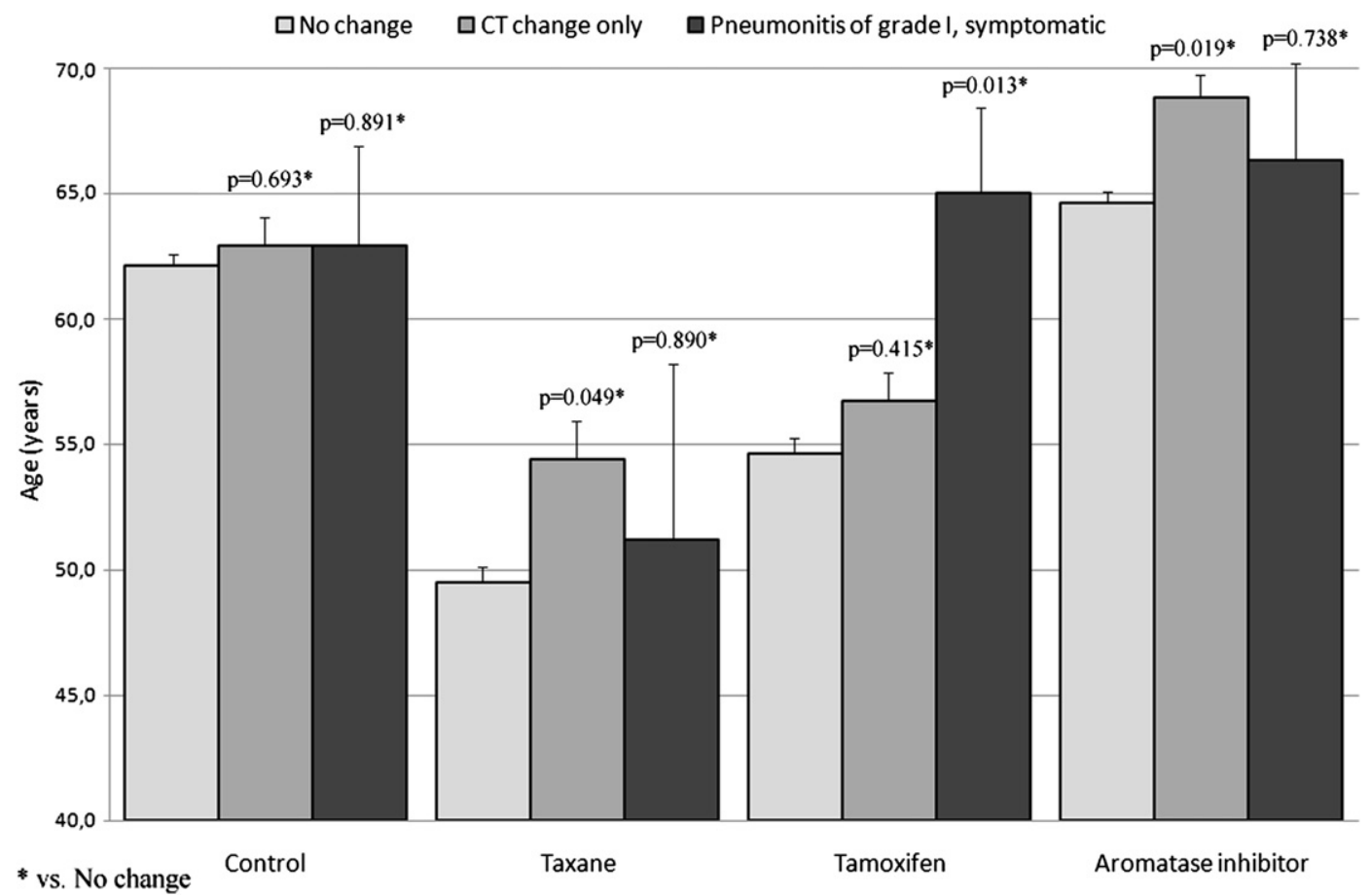

Fig. 1. Associations of Grade 1 pneumonitis and age (mean $\pm \mathrm{SE}$ ) with the various forms of systemic therapy.

The optimal sequencing of tamoxifen and radiotherapy has not yet been established. Three retrospective clinical studies were consistent in demonstrating no significant difference in outcome in terms of local control, relapse-free survival, and overall survival if tamoxifen was given concurrently or sequentially with radiotherapy (39). We beleive that, on the basis of the increased risk of radiation fibrosis and the lack of demonstrable therapeutic benefit if tamoxifen is used concomitantly with radiotherapy, their coadministration should be avoided.

The use of third-generation aromatase inhibitors is currently the standard endocrine therapy for postmenopausal women with hormone-dependent breast cancer (16). For this reason, we set out to test the effects of anastrozole and letrozole administered in conjunction with radiotherapy. Although estrogen deprivation could in theory exert a disadvantageous effect on postirradiation tissue remodelling (40), no change was observed in the risk of radiogenic lung sequelae. Our results accord with those of Azria and Ozsahin $(25,26)$, who found no association between the concomitant administration of letrozole with radiotherapy and the development of subcutaneous fibrosis. As far as we are aware, ours is the first well-powered study that has specifically pointed to lung complications and the use of aromatase inhibitors in the clinical radiotherapy setting.

The findings regarding the risk of radiation pneumonitis after chemotherapy are controversial. Radiation lung sequelae were found to be more frequent in breast cancer patients who received chemotherapy in some studies $(19,21)$, whereas in others no difference was seen $(14,23)$. The addition of taxanes to anthracyclin-based chemotherapy improved the survival in early breast cancer (30), and their use in clinical practice is universal. Early reports on the coadministration of paclitaxel with adjuvant radiotherapy suggested an increased risk of lung sequelae (27-29), whereas in a retrospective analysis of 189 breast cancer patients treated with radiotherapy and 5-fluorouracil-doxorubicincyclophosphamide chemotherapy, randomly with or without paclitaxel, Yu et al. (30) found equally low incidences of radiation pneumonitis and no difference between the two groups

Table 5. Multivariate analysis of the effects of age, MLD, and systemic therapy on early and late radiogenic lung sequelae

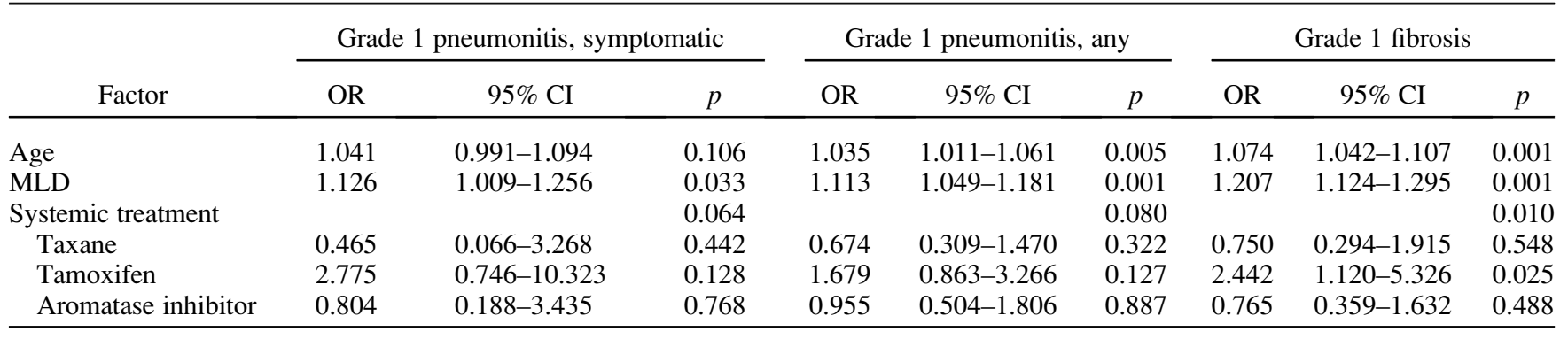

Abbreviations as in Tables 1 and 4. 


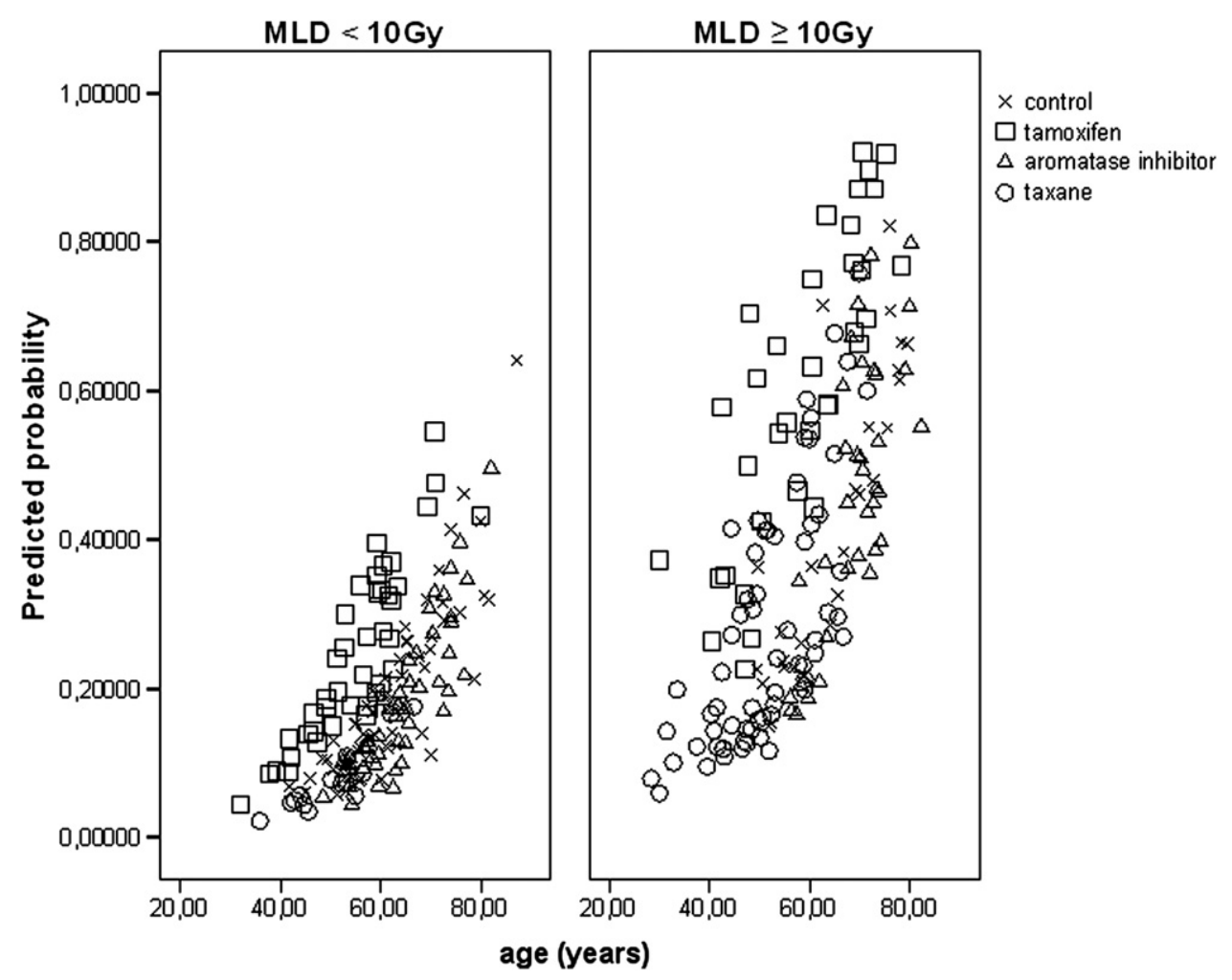

Fig. 2. Risk of Grade 1 radiation fibrosis as indicated by multivariate logistic regression model including patient age, radiation dose to the ipsilateral lung, and type of systemic treatment. Note that although in the model mean lung dose (MLD) was a continuous variable, in the graph for delineation, the median mean lung dose value of $10 \mathrm{~Gy}$ was used as a threshold.

(5\% vs. $4.5 \%$ ). In our study, the provision of chemotherapy involving paclitaxel or docetaxel was not associated with a higher risk of radiation pulmonary complications. In fact, despite the significantly higher irradiated lung volumes, the incidence of pulmonary toxicity was negligible. This finding can be explained in terms of the significantly younger age in the taxane group and is consistent with our results demonstrating the greatest influence of age on lung complications (13).

Age is one of the most important risk factors of radiation lung complications. Within the tamoxifen group, those patients who developed radiation pneumonitis were significantly older than those who did not (Fig. 1). The question arose as to whether tamoxifen treatment is in synergy with age, but our analysis did not support this. In the control and aromatase inhibitor groups, the radiogenic lung changes were not related to age, probably because of the lack of a similarly broad range of age as in the tamoxifen group.

\section{CONCLUSIONS}

The concomitant administration of tamoxifen with adjuvant radiotherapy independently increases the risk of radiation lung fibrosis, whereas the aromatase inhibitors and sequential taxane-based chemotherapy exhibit no such effect. Our results suggest that tamoxifen should not be administered during radiotherapy.

\section{REFERENCES}

1. Early Breast Cancer Trialists' Collaborative Group. Favourable and unfavourable effects of long-term survival of radiotherapy for early breast cancer: An overview of the randomised trials. Lancet 2000;355:1757-1771.

2. Cronin KA, Feuer EJ, Clarke LD, et al. Impact of adjuvant therapy and mammography on U.S. mortality from 1975 to 2000 . Comparison of mortality results from the CISNET Breast cancer base case analysis. J Natl Cancer Inst Monographs 2006;36:112-121.

3. Whelan TJ. Use of conventional radiation therapy as part of breast-conserving treatment. J Clin Oncol 2005;23:1718-1725.

4. Pierce LJ. The use of radiotherapy after mastectomy: A review of the literature. J Clin Oncol 2005;23:1706-1717.

5. Skoczylas JZ, Bentzen SM, Overgaard M, et al. Time course of radiological lung density changes after postmastectomy radiotherapy. Acta Oncol 2000;39:181-187.
6. Svane G, Rotstein S, Lax I. Influence of radiation therapy on lung tissue in breast cancer patients. CT-assessed density changes 4 years after completion of radiotherapy. Acta Oncol 1995;34:845-849.

7. Lind PA, Wennberg B, Gagliardi G, et al. Pulmonary complications following different radiotherapy techniques for breast cancer, and the association to irradiated lung volume and dose. Breast Cancer Res Treat 2001;68:199-210.

8. Tell R, Edgren MR, Sverrisdottir A, et al. Radiation-induced cell cycle response in lymphocytes is not related to clinical side-effects in breast cancer patients. Anticancer Res 2003;23:3077-3083.

9. Wennberg B, Gagliardi G, Sundbom L, et al. Early response of lung in breast cancer irradiation: Radiologic density changes measured by CT and symptomatic radiation pneumonitis. Int $J$ Radiat Oncol Biol Phys 2002;52:1196-1206. 
10. Tsougos I, Mavroidis P, Rajala J, et al. Evaluation of doseresponse models and parameters predicting radiation. Phys Med Biol 2005;50:3535-3554.

11. Ooi GC, Kwong DL, Ho JC, et al. Pulmonary sequelae of treatment for breast cancer: A prospective study. Int J Radiat Oncol Biol Phys 2001;50:411-419.

12. Kiricuta IC, Gotz U, Schwab F, et al. Target volume definition and target conformal irradiation technique for breast cancer patients. Acta Oncol 2000;39:429-436.

13. Kahán Z, Csenki M, Varga Z, et al. The risk of early and late lung sequelae after conformal radiotherapy in breast cancer patients. Int J Radiat Oncol Biol Phys 2007;68:673-681.

14. Gagliardi G, Bjohle J, Lax I, et al. Radiation pneumonitis after breast cancer irradiation: Analysis of the complication probability using the relative seriality model. Int J Radiat Oncol Biol Phys 2000;46:373-381.

15. Theuws JC, Kwa SL, Wagenaar AC, et al. Dose-effect relations for early local pulmonary injury after irradiation for malignant lymphoma and breast cancer. Radiother Oncol 1998;48:33-43.

16. Winer EP, Hudis C, Burstein HJ, et al. American Society of Clinical Oncology technology assessment on the use of aromatase inhibitors as adjuvant therapy for postmenopausal women with hormone receptor-positive breast cancer: status report 2004. J Clin Oncol 2005;23:619-629.

17. Carlson RW, Allred DC, Anderson BO, et al. Breast cancer. Clinical practice guidelines in oncology. J Natl Compr Canc Netw 2009;7:122-192.

18. Bentzen SM, Skoczylas JZ, Overgaard M, et al. Radiotherapyrelated lung fibrosis enhanced by tamoxifen. J Natl Cancer Inst 1996;88:918-922.

19. Koc M, Polat P, Suma S. Effects of tamoxifen on pulmonary fibrosis after cobalt-60 radiotherapy in breast cancer patients. Radiother Oncol 2002;64:171-175.

20. Huang EY, Wang CJ, Chen HC, et al. Multivariate analysis of pulmonary fibrosis after electron beam irradiation for postmastectomy chest wall and regional lymphatics: Evidence for nondosimetric factors. Radiother Oncol 2000;57:91-96.

21. Lind PA, Wennberg B, Gagliardi G, et al. ROC curves and evaluation of radiation-induced pulmonary toxicity in breast cancer. Int J Radiat Oncol Biol Phys 2006;64:765-770.

22. Harris EE, Christensen VJ, Hwang WT, et al. Impact of concurrent versus sequential tamoxifen with radiation therapy in earlystage breast cancer patients undergoing breast conservation treatment. J Clin Oncol 2005;23:11-16.

23. Holli K, Pitkanen M, Jarvenpaa R, et al. Early skin and lung reactions in breast cancer patients after radiotherapy: Prospective study. Radiother Oncol 2002;64:163-169.

24. Blom-Goldman U, Svane G, Wennberg B, et al. Quantitative assessment of lung density changes after 3-D radiotherapy for breast cancer. Acta Oncol 2007;46:187-193.

25. Azria D, Ozsahin M, Rosenstein B, et al. Multicenter phase II randomized trial evaluating toxicities of concurrent and sequential radiotherapy and letrozole (COHORT) as adjuvant therapy after conservative surgery in postmenopausal women with hormone receptor positive tumors: Preliminary results (Abstr.). Breast Cancer Res Treat 2007;106(Suppl.):144s.
26. Ozsahin M, Belkacemi Y, Rosenstein B, et al. Radiationinduced CD8 T-lymphocyte apoptosis yield predicts toxicities after adjuvant treatment with concurrent and sequential radiotherapy and letrozole in postmenopausal women with hormone receptor positive breast cancers: Preliminary results of the multicenter phase II randomized trial (COHORT) (Abstr.). Breast Cancer Res Treat 2007;106(Suppl.):166s.

27. Taghian AG, Assaad SI, Niemierko A, et al. Risk of pneumonitis in breast cancer patients treated with radiation therapy and combination chemotherapy with paclitaxel. J Natl Cancer Inst 2001;93:1806-1811.

28. Hanna YM, Baglan KL, Stromberg JS, et al. Acute and subacute toxicity associated with concurrent adjuvant radiation therapy and paclitaxel in primary breast cancer therapy. Breast $J$ 2002;8:149-153.

29. Burstein HJ, Bellon JR, Galper S, et al. Prospective evaluation of concurrent paclitaxel and radiation therapy after adjuvant doxorubicin and cyclophosphamide chemotherapy for Stage II or III breast cancer. Int J Radiat Oncol Biol Phys 2006;64: 496-504.

30. Yu TK, Whitman GJ, Thames HD, et al. Clinically relevant pneumonitis after sequential paclitaxel-based chemotherapy and radiotherapy in breast cancer patients. $J$ Natl Cancer Inst 2004:96:1676-1681.

31. Varga Z, Hideghéty K, Mező T, et al. Individual positioning: A comparative study of adjuvant breast radiotherapy in the prone versus supine position. Int J Radiat Oncol Biol Phys 2009;75: 94-100.

32. Azria D, Gourgou S, Sozzi WJ, et al. Concomitant use of tamoxifen with radiotherapy enhances subcutaneous breast fibrosis in hypersensitive patients. Br J Cancer 2004;91:1251-1260.

33. Anscher MS, Marks LB, Shafman DT, et al. Using transforming growth factor beta-1 during radiotherapy to select patients for dose escalation. J Clin Oncol 2001;19:3758-3765.

34. Li C, Wilson PB, Levine $\mathrm{E}$, et al. TGF- $\beta 1$ levels in pretreatment plasma identify breast cancer patients at risk of developing post-radiotherapy fibrosis. Int $J$ Cancer 1999;84: $155-159$.

35. Zujewski J, Pai L, Wakefield L, Giusti R, et al. Tamoxifen and fenretinide in women with metastatic breast cancer. Breast Cancer Res Treat 1999;57:277-283.

36. Border WA, Noble NA. Transforming growth factor $\beta$ in tissue fibrosis. N Engl J Med 1994;331:1286-1292.

37. Canney PA, Dean S. Transforming growth factor beta: A promotor of late connective tissue injury following radiotherapy? Br J Radiol 1990;63:620-623.

38. Schroth W, Goetz MP, Hamann U, et al. Association between CYP2D6 polymorphisms and outcomes among women with early stage breast cancer treated with tamoxifen. JAMA 2009; 302:1429-1436.

39. Harris EE, Christensen VJ, Hwang WT, et al. Impact of concurrent versus sequential tamoxifen with radiation therapy in earlystage breast cancer patients undergoing breast conservation treatment. J Clin Oncol 2005;23:11-16.

40. Calvin M. Oestrogens and wound healing. Maturitas 2000;34: 195-210. 